\title{
Smell's puzzling discrepancy: gifted discrimination, yet pitiful identification. ${ }^{1}$ (forthcoming) Mind and Language \\ Benjamin D. Young
}

\begin{abstract}
:
Humans are gifted at detecting and discriminating odors, yet we have difficulty identifying even the most prevalent everyday odors by name. This paper offers a new explanation for the puzzling discrepancy between our olfactory capacities for discrimination and identification by weaving together recent neuroscientific findings regarding the cortical connectivity of the olfactory system, the olfactory system's proprietary semantic integration center, and recent philosophical research on the olfactory system's compositional format of representation. The paper combines these areas of research to develop the comprehensive explanation that we cannot readily deploy conceptual resources in naming odorants, because of an incompatibility of compositional formats employed by our conceptual semantic resources and the olfactory system.
\end{abstract}

Keywords: Smell; Olfaction; Compositionality; Concepts; Non-Conceptual Content; Representational Format; Odor Identification; Odor Discrimination.

\footnotetext{
1 Portions of this paper have been presented at the Sierra Nevada Society for Neuroscience conference; CUNY, Graduate Center, Cognitive Science Symposium; The Hebrew University of Jerusalem's Cognitive Science Program; Queens College CUNY's Philosophy Department; the University of Haifa's Philosophy Department Colloquium; The Hebrew University of Jerusalem's Philosophy Department Colloquium; Ran Hassin's Lab Conscious in the Psychology Department at Hebrew University; The Cognitive and Information Science Group at UC Merced; Ben-Gurion University's Philosophy Department Colloquium; The Perception and Concept conference in Riga, Latvia; the European Society for Philosophy and Psychology's annual conference in Granada, Spain; at the workshop on Olfaction and Philosophy hosted by the Centre for Philosophical Psychology at the University of Antwerp; and at UNR's Philosophy Department WIPS, I would like to thank all of the members of the audience on these occasions for their helpful questions and feedback. I would especially like to thank Carlos Mariscal, Arnon Cahen, Hilla Jacobson, Jesse Prinz, and David Rosenthal for their comments on earlier versions of this paper. Additionally, I would like to thank all the reviewers whose feedback greatly improved the paper.
}

Address for correspondence:

Benjamin D. Young, The Department of Philosophy, University of Nevada, Reno 89557

Email: BenjaminYoung@unr.edu 


\section{Introduction}

Surprising as it may seem, humans are excellent smellers (Barwich, 2017; McGann, 2017). ${ }^{2}$ We are continually immersed in a sea of smells that shape our daily lives in a host of manners outside of our awareness. Olfactory perception influences mate selection, food preferences, kinship recognition, and social interactions. ${ }^{3}$ Moreover, the olfactory system is gifted in its capacity to discriminate and detect odors. ${ }^{4}$ The most recent estimate of our olfactory ability to discriminate odors places the number around one trillion (Bushdid et al, 2014). While this number and the model used to derive it has been criticized (Meister, 2015), the latest estimate of the dimensional space for olfactory qualities using perceptible discriminations exceeds that of vision and surpasses our ability to name odors (Keller, 2017). When considering our olfactory performance for detecting and discriminating odorants alone, we are gifted, yet a puzzling discrepancy arises when we compare this capacity to our ability to identify smells using names. English speakers are notoriously bad at naming odors to the extent that if it were not so pervasive across the population we might consider it pathological.

We are remarkably bad at naming odors that we encounter on a daily basis. As a means of self-demonstration Yeshurun and Sobel (2010) suggest performing the refrigerator smell test. Take any item out of the refrigerator and ask a blindfolded individual to name the item based on its smell. Most people cannot identify things as simple as peanut butter, ketchup, or mustard. Yet the refrigerator smell test is an unfair method for assessing our olfactory abilities, since these stimuli are being presented at a low temperature that is not optimal for generating the volatility of the chemical compounds necessary for the perception of olfactory quality. By comparison, the refrigerator smell test is on par with asking you to identify the colors of peoples' clothing in a dark movie theater. The do-it-at-home test is far from perfect, yet even under ideal conditions our ability to name odorants is poor.

In experimental settings humans can on average identify between $26 \%$ to $60 \%$ of the tested odorants by name, but this percentage varies across experiments, ages, and if the odorant is presented to both nostrils. In one of the earliest studies on odor identification, Cain (1979) showed that of the 80 familiar odors presented, participants could only identify $60 \% .^{5}$ In a further set of experiments Wijck \& Cain (1994) showed that the percentage of odors

\footnotetext{
2 An excellent overview of the cultural difference in olfactory abilities and how to improve olfactory perception can be found in Majid et al. (2017).

${ }^{3}$ Olfaction guides our food choices (Fallon and Rozin, 1983), dietary preferences (Rozin, 1978; Rozin, et al., 1986; E. Rozin, S. Rozin, and E. Rozin, 1992), our selection of mates (reviewed in Havlicek and Roberts, 2009; Young, 2014) and social acquaintances (Herz and Schooler, 2002; Jacob et al., 2002; Li et al., 2007), and is responsible for identification of kin (Russell, 1976; Porter, Cernoch, and McLaughlin, 1983; Porter, et al., 1986).

${ }^{4}$ Humans have two olfactory perceptual pathways, one from the front of the nose (orthonasal smell) and one from the back of the throat (retronasal smell). Retronasal smell is responsible for most of what we consider our experience of flavor. The paper concerns only orthonasal smell as the literature referenced on olfactory perception and its linguistic centers only concerns this pathway. Moreover, for the purposes of this paper all claims regarding the puzzling discrepancy are relativized to orthonasal smell. Additionally, English speakers display difficulty naming orthonasal smells, while identifying retronasal food odors seems easier. Readers interested in retronasal smell and flavor perception might want to look at Shepherd (2012) or for taste, at Smith (2007) or Lycan (2017).

${ }^{5}$ For a discussion of different methods of increasing olfactory encoding that yield increased descriptive accuracy in olfaction, but not necessarily identification, see Section 5 .
} 
correctly identified is related to age, with the highest rate of identification amongst young adults with $53 \%$ correctly identifying familiar odors. A follow-up study showed that odor identification varies with age - those aged 8-14 correctly identified 50\% of odorants, which increased to $60 \%$ in the 18-37-year-old cohort, but slowly dropped to $40 \%$ between the ages of 65-90 (Cain et al. 1995). However, these findings of 60\% accuracy at odor identification are rather high. Other studies have reported far lower rates of identification, such as $26.5 \%$ (Huisman \& Majid, 2018), 37\% (Distel \& Hudson), 29\% accuracy using one nostril, and 50\% using unfamiliar odors (Jonsson, Olsson, et al. 2005). One reason Cain et al's reported identification rates might be so high is that they use familiar odors from everyday life, but when unfamiliar odors are employed the accuracy of identification drops (Savic \& Bergulund, 2004). ${ }^{6}$ Additionally, Herz's (1999) findings that identification decreases with the use of only one nostril might explain the very low rate of identification in Jonsson, Olsson, et al. (2005). In a further set of experiments Jonsson et al (2005) showed that instances of failure of identification involve a failure of knowing (FOK) more than a tip-ofthe-tongue (TOT or TON tip-of-the-nose in olfaction) phenomena, which leads them to conclude that the lack of accuracy arises from a failure to identify the odor.

Failures of object identification are not unique to olfaction; we have similar issues with identifying unfamiliar faces, which is attributed to the representational format of facial representation being holistic. In fact, it has been suggested that odor identification might be similar to facial identification in that they both use holistic representational formats (Murphy et al. 1991). However, in a rather ingenious set of experiments, Stevenson and Mahmut (2013) show that odor identification is not completely analogous to facial identification. Even if we cannot identify a face, we still have access to associated semantic information about the face, yet if we fail to identify an odorant we cannot access semantically associate information about the smell. Stevenson and Mahmut conclude that we have fully formed odor percepts in these situations, yet there is poor connectivity between olfactory perceptual centers and semantic memory processing within olfaction. Thus, our failure in identification is directly linked to an inability to access semantic information about the target smell.

Herein we are presented with the puzzling discrepancy; ${ }^{7}$ humans are gifted at discriminating odorants, but pitiful at identifying odors by name. We can detect a vast quantity of odors (Bushdid et al. 2014) ${ }^{8}$ and the dimensionality of our capacity for olfactory

\footnotetext{
${ }^{6}$ In addition to the difference in accuracy at identifying odorants, Savic and Bergulund (2004) show that there are separate cortical areas responsible for processing familiar and unfamiliar odors.

7 The paper proceeds under the general assumption that our olfactory capacities for discrimination and identification are linked, thus by comparison there is a discrepancy between them. However, one could challenge this assumption and claim there is no puzzling discrepancy, rather we require two independent explanations: (1) what facilitates olfaction's superior discriminative ability for perceptual qualities when compared to other modalities; and (2) why are we bad at identifying smells by name. This alternative will not be entertained within the paper, as a more parsimonious and comprehensive single explanation can be provided assuming that capacities are linked. Both capacities and the discrepancy between them can be explained by noting the compositional encoding strategy at the sensory level of olfactory processing, which generates our efficient discriminative abilities, yet the representational format of some higher levels of olfactory processing, such as olfactory perception and cognition is incompatible with the format of semantic conceptual systems.

${ }_{8}$ Gerkin and Castro (2015) have disputed the methods used to generate this number and claim that the original study demonstrates that we can detect at most 1.72 trillion odors Olofsson. Even if their criticisms are accepted and the original model is corrected, the estimated number of odors humans can detect would still vastly exceed our ability to identity them, which would still yield the puzzling
} 
discrimination dwarfs that of vision (Keller, 2017), yet we accurately identify only a fraction of these olfactory objects (Engen and Ross, 1973; Cain, 1979). Identifying an odorant by name demands further cognitive resources than detection or discrimination, because it requires generating the sematic label of the olfactory stimulus, suggesting that our poor performance is linked to semantic processing issues (Jonsson et al 2005; Stevenson 2009; Veramendi et al 2013). As noted above, in the absence of a correct identification we cannot access associated semantic information about the odor (Stevenson \& Mahmut, 2013) indicating that the discrepancy arises as an interface issue between olfactory processing and our conceptual semantic resources. We cannot simply explain our poor accuracy at identifying odors as deriving from being bad at smelling, because quite the opposite is true. Olfaction is gifted in its capacity for detection and discrimination, yet frequently inaccurate when it comes to odor identification. How is it that we are so good at one and so bad at the other?

The paper develops a new explanation of this puzzle by showing that the olfactory system employs a system of compositionality that is incompatible with the compositionality required for the deployment of our semantic conceptual resources. The most basic conception of compositionality is that the meaning of a complex representation is derived from how it is composed from its constituent parts. However, it is easy to overlook that there are multiple types of compositional systems based on the different modes of composition employed in generating the complex representation. Thus, the thesis to be explored in this paper is that the difficulty in identifying odors by name derives from interconnections between cortical processing hubs that employ incompatible compositional formats. The explanation on offer builds upon recent work in contemporary chemosciences and neuroscience by arguing that we must note the type of representational format employed at each stage of processing in the olfactory system as it projects and interfaces with linguistic centers. What will be argued is that our puzzling olfactory abilities do not merely derive from our linguistic abilities or connectivity issues. Comparing the format employed in olfactory processing as it projects to linguistic centers with what is known about the representational format of concepts, generates a more comprehensive explanation of the puzzle than the current alternatives.

The proposed explanation that the discrepancy arises from a mismatch in compositional formats builds upon previous explanations by combining findings across levels of olfactory processing from sensory transduction, to perceptual states, and even cognitive states. To provide a more comprehensive explanation the paper unfolds across seven sections. Since research on the discrepancy between olfactory discrimination and identification has already been surveyed in the introduction, section 2 reviews previous explanations of our difficulty in identifying smells. Section 2 ends by introducing the thesis that the problem arises due to an incompatibility in representational formats. Explaining the discrepancy as an issue regarding incompatibility of compositional formats builds upon an unexplored aspect of Oloffson and Gottried's connectivity explanation, which is the closest competitor, as well as previous research arguing that the olfactory system employs a format of non-conceptual representations (Young, 2015). The new explanation shows its comprehensive strength by (a) surveying the representational formats across levels of olfactory processing, (b) combining findings across experimental methods, and (c) combining these data points to generate a parsimonious explanation of the discrepancy. Before surveying the olfactory literature, section 3 introduces compositionality, the different types of compositionality, and what is known about the cortical realization of compositionality. Section 3 not only provides

discrepancy. A detailed discussion of the vast dimensionality of odor quality space using judgments of just noticeable differences can be found in Young, Keller, and Rosenthal (2014) and Keller (2017). 
an introduction to compositionality under the assumption that the semantic concepts employed in naming tasks must minimally obey functional compositionality, but also introduces a more minimal form of non-decomposable functional compositionality that section 4 then shows is employed in olfactory processing. Section 4 provides evidence from olfactory sensory encoding, cortical processing, perceptual states, and cognitive states that olfaction employs the more minimal format of functional compositionality. Section 5 then considers a possible criticism that the discrepancy is culturally mediated and reflects Western societies' lack of a dedicated odor lexicon and dearth of daily conversation about smells. Finally, section 6 then concludes by noting future research that would help fully establish the theoretical explanation on offer.

\section{Previous Explanations of Our Olfactory Shortcomings}

Accounts of the puzzling discrepancy between our ability to discriminate and name odors have been offered based on the olfactory system's anatomy, functional organization, temporal transduction rate, and the effects of a culture's odor lexicon. ${ }^{9}$ However, rethinking the discrepancy between our olfactory capacities as an issue of representational format, creates an explanation that works in concert with aspects of these previous explanations in a coherent and integrated fashion.

\subsection{Attention and Temporal Transduction}

An intriguing explanation of our difficulty at identifying odors derives from the temporal processing of smells. Based on the slow transduction speed of the olfactory system, as compared to vision, it has been argued that we rarely attend to our olfactory sensory states. The lack of attention might make it more difficult for us to be conscious of our perception of olfactory stimuli (Sela and Sobel 2010). Thus, olfactory perception might be in a constant state of change blindness, whereby we simply cannot attend to the variegated nature of the odor plume continually traversing our nostrils, causing our difficulty in identifying odors. Continuing the analogy to vision is a similar explanation that argues olfactory perception is similar to Blindsight (Lorig 1989; Castro and Seeley 2014). Individuals with Blindsight can employ visual information, yet they are unable to semantically identify the visual stimuli, which resembles the puzzling discrepancy in our olfactory abilities.

Aside from the tyranny of holding the olfactory system hostage to the temporal processing speed of vision, the knock-on effect of slow stimuli transduction on attention might only play a small part in explaining the puzzling discrepancy. Despite olfaction's slow transduction rate, we can increase our conscious perceptual abilities simply by increasing exposure to odors and the number of our olfactory experiences. For instance, the primary means of perceptual and cognitive enhancement for olfactory recognition is not semantically mediated by increasing our attention or lexical prowess, but rather by increasing the amount and type of exposure to the olfactory stimuli (for a review cf Young, Keller, and Rosenthal, 2014; and Majid et al 2017).

Furthermore, following multiple studies on this olfactory discrepancy, it is widely accepted that the problem arises from a deficit in accessing semantic information about odors (Jonsson et al 2005; Stevenson 2009; Veramendi et al 2013). Further support for the claim that the problem arises from a difficulty at the interface between linguistic and olfactory information is derived from the tip-of-nose phenomena whereby we can recognize an odor

\footnotetext{
${ }_{9}$ The effect of cultural mediation on olfactory naming ability will be dealt with in the criticism section 5 .
} 
as a common everyday smell and be able to describe its usage, yet not be able to name it (Sulmont-Rose 2005; Gilbert, 2008; Keller, 2017). Consequently, the olfactory system's slow transduction might yield a general lack of conscious awareness of the changing stream of odors transiting our nostrils, yet slow temporal processing speeds are unlikely to explain the deficit and attention might only play a small mediating role. ${ }^{10}$

\subsection{Anatomical Explanations}

Another research paradigm is to sniff out an explanation of our odor-naming deficit by exploring olfaction's unique anatomical structures, cortical architecture, and neurofunctional connectivity. Currently there are three plausible explanations involving: the thalamus, the lateralization of olfactory processing, and cortical connections between olfactory sensory processing and cortical centers for semantic integration. ${ }^{11}$

\subsubsection{Thalamic Mediation}

The lack of a direct thalamic mediation between olfactory perceptual states and the olfactory cortex (reviewed in Young, 2012) has been put forward as one of the possible explanations of the poor connection between language and olfaction (Herz 2005). Initial research suggests that in general, thalamic connectivity plays an important role in language processing (Klostermann, et al., 2013). The thalamus's primary function in language processing might be attributed to its placement as a hub for interconnecting language centers (Crosson, 2013). To reiterate a claim stressed in the introduction, an explanation of our deficit at odor identification should avail itself of research on the representational formats employed at each anatomical hub and stage of processing. Empirical research conducted after Herz posed her (2005) explanation indicates that the functional role of the thalamus in language processing is the segregation of informational streams in language processing required to generate the compositionality of language (Theofanopoulou et al., 2016). The thalamus is claimed to play an integral role in synchronizing separate areas within linguistic processing as a necessary element in generating the compositional compounds of combinatorial linguistic representations within the brain. Even if the olfactory system does not have the same anatomical connectivity it might be supplanted with a functional analogue, such as the linguistic olfactory integration centers revealed by Olofsson et al., $(2013,2014)$ (see section 2.2.3). Since a functionally analogous realizer could perform the same role for olfaction, the mere lack of thalamic connectivity in olfaction cannot be the whole explanation.

\subsubsection{Lateralization}

The lateralization of semantic and olfactory processing serves as the basis for another possible explanation of the puzzle. Royet and Plailly, 2004 claimed olfactory processing occurs predominately within the right hemisphere while language processing primarily occurs in the left hemisphere (Bind et al., 2004). The lack of convergent processing within a

\footnotetext{
${ }^{10}$ A further reason to reject this explanation can be derived from research covered in section 5 showing that perfumers and coffee experts with an increased odor lexicon and daily attention to smells show the same deficit in identifying odors by name.

${ }^{11}$ An alternative fourth suggestion is that the difficulty in deploying semantic resources for olfactory identification is attributable to a cortical bottleneck based on competition for the same cortical resources (Lorig, 1999; Lorig, Elmes at al 1998). However, this explanation is left aside in what follows, as it is not substantiated by current research that shows olfaction has its own cortical center for semantic integration and processing.
} 
hemisphere might explain why it is so hard to deploy semantic resources for olfactory identification using semantic labels. Yet meta-analysis of published data from neuroimaging of the human olfactory cortex does not strongly support the hypothesis of cortical lateralization (Seubert et al., 2013). Moreover, the explanation assumes that lateralization is sufficient for generating a limited interaction between semantic and olfactory centers of cortical processing. Given the connectivity between hemispheres via the corpus collosum, this explanation would at best predict a lag in processing times for naming, but not necessarily a failure in accessing semantic content. ${ }^{12}$

On purely theoretical grounds, a counter-example from music comprehension and song identification makes this explanation highly implausible. The auditory encoding of musical stimuli occurs in the right hemisphere (Hendrick et al., 2014), while semantic linguistic processing occurs in the left hemisphere. Nevertheless, in tasks of identifying songs using either their title or melody the results of using either of these means of identification were the same (Zehra et al., 2008). Thus, hemispheric lateralization is not sufficient to explain our poor naming ability of odors, as an analogous deficit is not found within the lateralization of auditory music stimuli and linguistic tags.

\subsubsection{Cortical Connections}

A more recent line of research explains the discrepancy between semantic resources and olfactory processing as a connectivity issue. In a progressive series of experiments, Olofsson and Gottfried (2013, 2014, 2015), mapped the linguistic centers responsible for our ability to linguistically represent and verbally report our experience of odors. Identifying and naming a stimulus requires a three part series of perceptually transducing the odor object, representing the stimulus, and generating a verbal report using the representation of the perceptual entity. Using this tripartite breakdown of the prerequisites of fulfilling a naming task, they began by first studying the verbal production of semantic tags for odors. Using a cohort of patients suffering from primary progressive aphasis (PPA) Olofsson and Gottfried (2013) localized areas within the temporal pole (TP) and inferior frontal gyrus (IFG), which process the verbalization of odors. In a further set of studies, they showed that the temporal pole in the anterior temporal lobe (ATL) mediates the connection between olfactory representation in the piriform cortex and language centers (Olofsson et al 2014). Based on the sequence of studies they identify these areas as olfactory specific centers for olfactory and semantic integration, which are dedicated to the linguistic mediation of olfactory representations.

Employing these studies, they note that there are only three relays between olfactory sensory processing and these cortical areas. The lack of connectivity leads Olofsson and Gottfried to hypothesize that the lack of cortical relays yields a dearth of processing. Accordingly, they conclude that olfactory semantic integration is inferior, because less processing has occurred to the sensory information by the point that it reaches the ATL (Olofsson et al 2015). Their theory tacitly assumes that the decreased cortical connectivity implies a decreased amount of processing and this alone is sufficient to explain the puzzling discrepancy. ${ }^{13}$ While their research is fundamental in unraveling our perplexing olfactory

${ }^{12}$ I must thank two anonymous reviewers for suggestions that sharpened these criticisms of the lateralization explanation.

${ }^{13}$ Keller (2017) accepts the line of argument that insufficient connectivity to language centers sufficiently explains the discrepancy between our naming and discriminative abilities, but claims this is in keeping with the overall function of olfactory perception. His argument is that olfaction is selectively designed for the guidance of action and behavior, thus it has greater connectivity with 
abilities, there is a significantly under explored aspect of their proposal concerning the format of olfactory representations, which I will seek to complete. Building upon previous research that olfaction employs a different form of compositionality than concepts (Young, 2015), this paper develops the argument that it is not just the amount of connectivity and processing that matters, rather that understanding the representational format at each level of processing is required to explain the puzzle. The moves of this argument proceed through the next few section as follows: Section 2.3 introduces the representational format explanation, section 3 reviews the different types of compositionality including in section 3.3 a discussion of the role of the ATL in generating the representational format of compositionality; section 4 then wraps up the argument by surveying research across each level of olfactory processing indicating that the olfactory system implements a different compositional format.

\subsection{The alternative - Representational Format}

Our inability to identify smells by name is not only attributable to the lack of connections between olfactory processing and language centers; rather the location of these interconnections allows us to infer that the best explanation involves interface issues derived from an incompatibility of formats. The cortical connection that feeds into the olfactory language center identified by Olofsson et al., (2013, 2014) derives from the piriform cortex (PC), whose encoding structure is distributed in a manner that is not reminiscent of the compositionality observed in the other perceptual systems. ${ }^{14}$ Thus, it is not just the connectivity, but also an incompatibility of representational formats that explains the puzzling discrepancy.

Since our deficit for naming odors arises from how olfactory processing accesses semantic resources (see Introduction and section 2.1) it will be assumed that identification, as assessed by naming tasks, requires the deployment of semantic conceptual resources and this is where problems arises. Section 3 develops the idea that concepts employ a representational structure that is compositionally formatted. The section offers a brief introduction to compositionality and the neural realization of the compositionality of concepts. Following this overview, section 4 offers evidence that the olfactory system projecting to the ATL and some of our experiences of complex smells do not employ this same form of compositionality. Thus, a more comprehensive explanation can be provided by showing that olfaction and our conceptual semantic resources employ different compositional formats.

cortical areas responsible for these functions. Nonetheless his account does not offer an explanation of why the lack of connectivity on its own is sufficient for explaining the discrepancy. Moreover, it is unclear how his account can handle the cultural differences in olfactory naming abilities (see section

5) without a risk of confabulating the selected function of olfaction with its current function.

${ }_{14}$ The alternative explanation offered in this paper derives from both the olfactory system's interconnection with language centers and the format of representations employed in processing at these hubs. Explaining the puzzling discrepancy in a comprehensive manner requires considering the olfactory pathways, cortical connections, and representational formats at each stage of processing. In an ideal hypothetical system everything and anything can be connected and processed along a host of different pathways and formats. However, using olfaction as the model system in this instance constrains the realm of possibilities. For instance, the constituents of compositional odor representations cannot simply be decomposed and inputted into processing by the conceptual cortical systems, because of a lack of direct and unmediated pathway between these processing areas. In fact, even when complex mixtures yield elemental mixtures (see section 4.2) this is subserved by independent olfactory pathways in the amygdala. 


\section{Compositionality and Concepts}

The research surveyed in the introduction indicates that the difficulty in identifying odors by names arises in accessing semantic resources. Given this diagnosis this section turns to analyzing the compositional format of concepts, because as argued elsewhere, (Young, 2015) even at their most minimal conception, concepts are the components of semantically meaningful thoughts. By first looking at the compositional format of concepts we can then compare this representational format to that employed by olfaction in support of the thesis that puzzling deficit arises from an incompatibility in representation format between the olfactory and conceptual semantic processing centers.

Despite much disagreement in both Philosophy and Cognitive Science regarding the nature of concepts, there is consensus that concepts are to some extent compositional (Jylkka, 2011; Piantadosi et al. 2016; Connell \& Lynott, 2010; Del Pinal, 2015; Frixione, \& Lieot, 2012). Theories of concepts vary widely on the details of how our conceptual system implements and respects the notion of compositionality. Despite these differences it is traditionally assumed that one of the essential characteristics of concepts is that they are minimally sensitive to and have access to their constituents.

There is a long-standing tradition of inferring the representational structure of our cognitive states from our cognitive or perceptual capacities. Most famously this move is seen in the systematicity argument where the compositional syntax of thought is inferred from the compositionality of language (Fodor, 1987, Fodor \& Pylyshyn 1988, Fodor \& McLaughlin 1990). However, this strategy is also found in the productivity argument (Fodor, 1981, 1985, 1987, Fodor \& Pylyshyn 1988), visual and auditory systematicity (Cummins, et. al 2001), the tracking argument (Horgan \& Tienson, 1994, 1996, 1997), and our capacity for inferential coherence (Crane, 1990; Fodor, 1985; Horgan \& Tienson, 1994, 1996). Our mental economy's systematic and productive nature together with the stability of conceptual content, intra-subjectively across time and inter-subjectively at any instance, requires that our conceptual systems must be compositional. In what follows, I shall remain neutral regarding the nature of concepts and only assume the minimal consensus for the sake of argument that whatever concepts might be, they must obey compositionality to some extent. (Young, 2015).

Compositionality is determined relative to a system of representations, such that it requires specifying the primitives for the system, the rules of formation and transformation, as well as the rules of well-formedness allowed by the system for generating meaningful complex expressions (van Gelder, 1990). The minimal conception of compositionality requires a specification of the interrelation between the syntax and semantics of a system of representation, such that the meaning of a complex expression is determined by the meanings of the atomistic parts and the systems mode of combination (Dever, 2005, 2012, Pagin and Westershtal 2010a-b). The most important factor in determining whether a system is compositional is the mode of combination i.e. the rules governing the composition of complex expressions. The mode of combination determines whether a system is compositional, as well as the type of compositionality.

Intuitively we assume the classical conception of compositionality as requiring part whole relations, whereby the complex representation syntactically contains the constituents. Or to put it more succinctly, our intuitive assumption is that any representational system that implements compositionality must do so via concatenation. The section proceeds by introducing the strong classical conception of concatenative compositionality, which will be contrasted with the less stringent notion of functional compositionality. Section 3.2 
introduces a more minimal form of non-decomposable functional compositionality, which is implemented by the olfactory system. Section 3.3 concludes the discussion of compositionality with a brief overview of what we know about the neural realization of the compositionality of concepts, in relation to the cortical hubs of olfactory language processing.

\subsection{Concepts and their relation to Classical (Concatenative) Compositionality}

The most stringent form of compositionality occurs in Fodor's Language of Thought Hypothesis (LoTH) (1976, 1981, 1987, 2000, 2008), according to which, thought occurs in a representational medium similar to language, such that each thought is the concatenation of its constituents. ${ }^{15}$ According to the LoTH all thoughts must occur in such a compositional rubric as evidenced by the systematic and productive nature of our cognitive and linguistic abilities (Fodor, 1981, 1987; Fodor \& Pylyshyn, 1988; Fodor \& McLaughlin, 1990). Classical compositionality is merely concatenation based on the spatial juxtaposition of expressions within the complex mental representation. Simply put, the complex representation must have as a proper part an instance of each of the constituents. The internal structure of the system's states must be of a formal nature, such that the constituency relations among the expressions are directly mirrored in the structure of the corresponding tokens. Thus, the explicit syntactic representation of the primitives within the complex mental representation is required. While concatenative compositionality is a rather extreme form of compositionality, it is quite clear that Fodorian LoT requires such a robust format (Dever, 2005, 2012; Pagin and Westersthal, 2010a). However, the applicability of concatenative compositionality to concepts and thought is questionable (Clapp, 2012). Furthermore, the theory of concepts generated from this concatenative standpoint is far from widely accepted in the cognitive sciences.

With the rise of research on our psychological conceptual capacities, their inferential roles, and their biological realization, it has become clear that while concepts require compositionality, how they implement it varies from the logical part-whole concatenation espoused by the LoTH. Contemporary views of concepts still hold compositionality dear, yet their means of implementation can vary drastically. There are even those who still espouse the strong form of classical compositionality while arguing that it can be realized in non-classical systems.

Prototype theories of concepts stand in stark contrast to the atomistic theories of concepts predicated upon a concatenative internal syntactic structure. According to prototype theory (and most statistical regularity theories of concepts) the complex structure of a concept encodes a statistical typicality relation of the properties their members tend to have (Laurence and Margolis, 1999, p. 27; Murphy, 2002). ${ }^{16}$ Recognizing the challenge of generating compositionality within these systems (Fodor, 1981; Fodor and Lepore, 1996) prototype theories have offered various implementation strategies for accomplishing classical compositionality (Prinz, 2002, 2012; Del Pinal, 2015; Frixione and Lieot, 2012).

\footnotetext{
${ }_{15}$ Versions of concatenative compositionality can also be found in Frege (Szabo 2000; Dever 2006) who endorses a version of mereological composition according to which the meaning of the parts of the sentence are explicit within the meaning of the entire sentence. In contemporary philosophy of language Jacobson (2002) and Barker and Jacobson (2007) have defended strong direct compositionality that is equivalent to concatenative compositionality (also referred to as strong parallelism) (Szabo 2000a-b; Dever, 2006).

${ }_{16}$ The current construal of prototype theory is a simplification that is adequate for purposes of this paper, but it does not do justice to the nuances between different versions of prototype theory.
} 
However, more scientifically plausible and biologically implementable theories have been offered that create compositional concepts not in terms of concatenative representations. These transitional theories still maintain that complex concepts require constituent structures to be compositional. However, they weaken the requirements for generating constituent structures, either by employing a multivariate system that respects classical compositionality using biologically plausible versions of tensor product networks, (Steward and Eliasmith, (2012) or by allowing that the constituents do not need to be literal components of the complex representation (Rice, 2013).

What unites the aforementioned theories of concepts is their adherence to the necessary condition that compositional representations require internal constituents or be connected via composition or decomposition functions to the relevant constitutive concepts. However, it might be possible to realize compositionality in non-concatenative representational systems either using a dual layer theory such as the probabilistic language of thought (PLOT) (Goodman et al. 2015) or by assuming a hierarchy of flexible distributed representations comprised of modality specific conceptual features (Kiefer \& Pulvermuller, 2012). Contemporary biologically plausible theories of concepts wishing to maintain their allegiance to compositionality without the strictures of concatenative compositionality might avail themselves to the more permissive format of functional compositionality.

\subsection{Functional Compositionality}

Minimally, all that is required for a system of representations to be considered compositional is the specification of a relation for the combination of expression types and primitives. Recognizing the theoretical possibility of a more minimal form of compositionality, van Gelder proposed functional compositionality, which requires a systematic manner for creating compound expressions given the constituents, and a similar systematic mode for decomposing the complex representations into their constituents (van Gelder, 1990). Mental representations with functional constituent structures are formed from the constituents and can be decomposed back into them, but these complex representations do not have constituent parts which we can refer to as their internal structure.

van Gelder's theoretical motivation for formulating compositional representations without discrete syntactic constituents was to explain how connectionist networks could generate systematicity. For this reason, he thought it was of grave importance to show that the system could both represent the complex representation $a R b$ and it's systematic variant $b R a{ }^{17}$ With systematicity as van Gelder's target phenomena, his proposed theoretical functionally compositional system required reliable rules of processing for generating the complex from the constituents, decomposing these functionally complex representations, and transforming these same constituents into systematic variants (Martinez-Manrique, 2014, p. 309). For van Gelder's purposes the decomposition function plays an integral role and is a necessary condition for a representational system to be functionally compositional (van Gelder, 1990, p. 361).

However, a more minimal form of compositionality is possible that jettisons the ability of the system to functionally decompose the complex representation into its constituents. The

${ }^{17}$ For present purposes I am assuming Cummins et al. (2001) neutral interpretation of systematicity, which is less contentious for connectionist theories than the linguistic examples of systematicity often stated by LoT theorists. 
most minimal form of compositionality does not require that the components persist within the complex expression or that the complex be decomposable into its previous component parts, but only that the meaning of the whole is determined from the meaning of these parts. ${ }^{18}$ The functionally complex representation is endowed with complex meaning within the system, by virtue of being formed from constituents. Thus, it is a theoretical possibility that a complex representation with constituent structure need not require an internal syntactic structure of the classical type, nor that it be decomposable back into these constituents.

The minimal conception of compositionality allows for a permissive stance on compositionality that only requires the complex representation be functionally composed from the constituents. ${ }^{19}$ Our olfactory sensory, perceptual, and cognitive states can be used to establish that the olfactory system employs this minimal non-decomposable form of functional compositionality. ${ }^{20}$ The mismatch of format explains why it is that we cannot readily identify smells, because the semantic conceptual system employs a different type of compositionality than the olfactory perceptual system. Before surveying the evidence indicating that the olfactory system implements non-decomposable functional compositionality, the next section covers the cortical realization of complex compositional representation of concepts in general, as well as in connection with what is known about the ATL, which was identified in section 2.2.3 as the center of olfactory and semantic integration.

\subsection{The Neural Realization of the Compositionality of Concepts}

Most theories of concepts agree that concepts must be sensitive to the creative, productive, systematic, and stable nature of our cognitive states. Our capacity to think thoughts with complex semantic content derives from our inferential sensitivity to a hierarchy of semantic content and rich interrelations between the associated constituents that generate the complexity we attribute to concepts. What is decisive throughout the literature on concepts is that these entities either have an internal structure that can be used to explain their complex semantic value or we can refer to their constituents by way of their compositional formation and decomposition function. Regardless of ones' theory of concepts, a necessary

\footnotetext{
${ }_{18}$ Non-decomposable functionally compositional representations are compatible with Dever's (2005, 2012) minimal compositionality that is syntactically functional, yet non-concatenative. "Functionally, compositionality is a property had by a meaning theory for a language just in case, for any complex expression in the language, its meaning is some function of the meanings of its component parts and their mode of syntactic organization" (Dever, 2012, p. 93).

19 Contrary to this permissive understanding of the theoretical possibility of functional compositionality, Szabo (2000a, 2000b) has challenged the adequacy of functional compositionality in relation to mathematics. However, a review of empirical evidence regarding the non-conceptual format of olfaction (Young, 2015) provides reason to think that a form of functional compositionality is not only theoretically viable outside of the domain of mathematics, but actually biologically implemented in olfaction.

20 While some might object that this methodology conflates separate levels of explanation and analysis, as well as riding roughshod over the personal subpersonal distinction, in keeping with representational pluralism (Cummins et al., 1996, 2001) and non-conceptual pluralism (Bermudez, 2007) I am attempting to be as neutral as possible regarding the correct level of analysis for mental content, concepts, and non-conceptual content. Moreover, given that the debate regarding the nature of concepts and mental representation is so variegated in endorsements of favored levels of ontological or epistemic analysis, for present purposes I find the personal subpersonal distinction of little use other than for obfuscation. Additionally, even if a theorist chooses to disregard the stimuli encoding and neural transduction evidence covered in throughout sections 4.1-4.1.2 my argument can still be substantiated with the evidence offered in sections 4.2-4.3.
} 
feature of concepthood is that we can potentially access the constituents that compose the complex representation when deploying the concept.

Only recently have we begun to identify the underlying cortical realization of the hierarchical structure required to implement these notions of conceptual compositionality. The cortical realization of concepts is accomplished through dynamic hierarchical binding across levels of neural networks, which generates coherent internally structured complex representations without an explicit concatenative syntax (Maye \& Engel, 2012). Furthermore, the perception of visual objects depends upon hierarchically structured semantic resources. Visual object perception is refined using these high-level object features that are coded together with semantic representations (Clarke et al., 2013, 2015). These studies provide strong evidence that hierarchically structured semantic information plays an integral role in the formation of visual object representations with their paradigmatic compositional format. The aforementioned results might be relativized to vision, but there are similar experimental results for the cortical realization of the hierarchy of compositional structure in semantic linguistic processing.

Parts of the anterior temporal lobe (ATL) are key in processing conceptual representations (Tyler et al. 2004, 2013; Moss et al. 2005; Patterson et al. 2007; Lambon Ralph et al. 2010; Mion et al. 2010; Clarke and Tyler 2014; Clarke et al., 2015; Binder, 2016), which is in keeping with Olofsson et al.'s (2013, 2014) research on the semantic processing areas of olfaction. However, the ATL's role in linguistic processing involves representing the syntactic organization and hierarchy of linguistic stimuli, while the Angular Gyrus (AG) encodes the semantic features and hierarchy that we would commonly refer to in explaining compositional thought. A series of studies have shown that the AG processes combinatorial semantics (Humphries et al., 2006), forms the neuroanatomical hub for conceptual combination (Price et al., 2015), and plays a causal role in the compositional integration of lexical semantic information (Price et al., 2016). However, all three studies indicate that the ATL does not process the combinatorial features of semantic processing required in implementing the semantic hierarchy that we attribute to the compositionality of concepts. Not only has it been shown that the cortical realization of conceptual sematic capacities requires a hierarchical structure of encoding allowing for compositionally complex semantic concepts that are sensitive to their individual constituents, but also that the ATL does not encode the semantic aspects of compositional representations for conceptual representations.

\section{Functionally Compositional Olfactory Representations}

Olfactory processing does not always employ the same form of compositionality as concepts, which explains the difficulty of interfacing with semantic conceptual resources in naming identification tasks. Olfactory states are formatted in a manner that is mediated more by sensory templates than by our conceptual repertoire and lexical resources (Young, Keller, and Rosenthal, 2014). This section explores the compositional format that mediates olfactory encoding of odorants projecting to the olfactory language processing centers in the ATL. Additionally, this section explores the available content of olfactory experiential states at the level of perception and cognition.

Prima facie, olfactory experiences occur in a different fashion than states mediated by our semantic conceptual resources. While olfactory states are arguably representational, ${ }^{21}$ the

${ }^{21}$ Arguably it is the case that olfactory experiences have representational content, because of cases of olfactory misrepresentation (misidentification and misattribution of the olfactory object), the 
structural nature of the representational system employed by olfaction is combinatorial, but not concatenatively compositional (Young, 2015) or functionally compositional in the stronger sense. The section proceeds by extrapolating the compositional structure of olfactory states from olfactory sensory transduction, perceptual encoding, and cognitive states.

\subsection{Stimuli Transduction and Syntactic Encoding}

The most striking difference between olfaction and the other sensory systems is the lack of isomorphism between receptor types and perceptual stimuli. There is no strict chemotopic mapping of chemical properties of the distal stimuli to individual receptor types or higherlevel glomeruli in the olfactory bulb. The lack of chemotopic maps either at the receptors or olfactory bulb is quite unlike vision with its retinotopic maps and orientation columns in $\mathrm{V} 1$, or audition with the decomposition of frequency in the cochlea. Furthermore, olfactory cortical states encode complex odor compounds not simply as the summation of their constituents, which begins to provide the basis for an explanation of our inability to perceptually identify more than four components of complex olfactory mixtures (section 4.2), which arguably shows that olfactory perceptual experiences are functionally compositional in the weak non-decomposable sense.

\subsubsection{Olfactory Receptor Neurons and the Olfactory Bulb}

Odorants are transduced in a combinatorial manner (Buck and Axel, 1991; Malnic et al. 1999; Araneda et al. 2000; Firestein, 2001; Meierhenrich et al. 2004; Hallem et al. 2006a, 2006b). At the initial sensory level, the olfactory system encodes the molecular structure of chemical stimuli in a distributed fashion across multiple olfactory receptor neurons $(O R N)$. Odorants are combinatorally encoded in a spatially extended and parallel fashion across multiple receptors, both at the receptor level and at the further stage of processing within the olfactory bulb. The glomeruli and mitral cells within the olfactory bulb $(O B)$ encode input from across multiple ORNs. Glomeruli show a preferential firing pattern for particular chemical structures but are also sensitive to other chemical stimuli. As a result, a chemotopic map does not arise within the glomeruli of the olfactory bulb. The olfactory bulb might not have a strict chemotopic map like the retinotopic map of vision, but it does have a coarse chemotopic organization (Bozza \& Mombaerts, 2001).

The combinatorial encoding of a stimulus is distributed across multiple regions throughout the glomeruli of the $O B$, with each glomerulus being sensitive to different parts and combinations of the chemical structure. Odorant encoding occurs in a parallel and distributed manner across multiple glomeruli. The distributed nature of olfactory stimulus encoding within the olfactory bulb suggests that monomolecular, and by extension complex odors are not encoded as the sum of their parts, rather they have their own unique distributed patterns of activation (for a review cf Auffarth, 2013). In addition to the spatially

intentional inexistence of the olfactory object, and our ability to represent olfactory experiences under different conceptual guises (representing as $\mathrm{x}$ ) (Young, 2011). Further arguments for the representational status of olfactory experience can be found in Batty (2009, 2010a-c) and Lycan (2014). However, the stimulus driven approach favored by the aforementioned theories has been criticized by Barwich $(2014,2017)$ who proposes a process based theory of olfactory perception, and Keller $(2015,2017)$ who endorses the non-objective process view. The paper cannot do justice to this debate as it diverges into a larger debate regarding how to conceive of the olfactory object, but the explanation on hand as an expansion of the cortical connectivity explanation (section 2.2.3) falls within the overarching research program endorsed by Olofsson and Gottfried (2015) that we should treat smells as objective entities. 
distributed encoding of olfactory stimuli across glomeruli, the encoding dynamic is also temporally extended (Linster \& Cleland, 2013, Olofsson, 2014; Hadad et al 2014; Sanders et al 2014). Olfactory stimulus encoding is accomplished by large groups of glomeruli and mitral cells firing across time, such that stimuli transduction is spatially and temporally distributed. ${ }^{22}$

The distributed nature of the spatiotemporal structure of sensory representations might be used to adjudicate against concatenative representations with their strict internal syntax. Given that the primitives within a concatenative compositional system are literal parts tokened within the complex expression, it is prima facie not obvious how this could be accomplished by the olfactory bulb's encoding of odorants. Since complex olfactory mixtures are encoded in a spatiotemporally parallel and distributed fashion within the OB, literally tokening the constituents is not possible at a given time. However, relaxing the synchronic condition of compositionality to allow for distributed temporal parts would still not circumvent the problem, as they would still be spatially distributed in a manner unlike any example that has formal parts within a complex representation. Hence, olfactory stimuli at the sensory level of olfactory processing are not encoded by a concatenative syntactic system.

Sensory transduction and olfactory stimuli encoding provide strong evidence that olfaction implements a syntactic system employing functional compositionality. However, this does not yet show that olfaction implements a form of compositionality incompatible with all formats of concepts. What needs to be further demonstrated is that odorant encoding forms complex representations that generate compositionality that does not allow access to the constituents or that cannot be decomposed.

\subsubsection{Odorant Encoding at the Piriform Cortex}

The rough chemotopic organization found within the olfactory bulb is not maintained in the olfactory systems projections to the Piriform Cortex $(P C) .{ }^{23}$ In animal studies of the neural encoding of odorants it has been shown that the $P C$ does not maintain the chemotopic organization found in the $O B$. Rather, convergent neural ensembles respond to dissimilar molecular structures of odorants in the same way (Stettler and Axel, 2009). This is in marked contrast to the visual, auditory, and somatosensory systems that preserve the spatially organized transduction of the similarity of stimuli features from the sense organ through their respective sensory cortexes (Woolsey and Walzl, 1942; Talbot and Marshall, 1941; Marshall et al., 1941). Moreover, odorant encoding within the $P C$ is represented in a sparse and spatially distributed fashion that does not code the minute differences between the structural components within complex odor mixtures (Illig \& Haberly, 2003; Wilson \& Stevenson, 2006; Rennaker et al, 2007; Stettler \& Axel 2009). ${ }^{24}$ Additionally, recent

${ }^{22}$ Research by Johnson et al. $(2007,2010)$ has questioned the role of temporal processing time. Their research indicated that odorants are combinatorally encoded in a spatial and non-summative (nonconcatenative) manner, but temporal distribution patterns are not required for odor encoding. However, Hadad et al (2014) refuted these their claims by showing that the olfactory bulb is sensitive to temporal dynamics for odorant encoding, while the PC is less sensitive to these temporal dynamics and more selectively sensitive to the neural firing rates outputted from the olfactory bulb. ${ }_{23}$ An excellent account of the debate regarding the role of the Piriform Cortex in olfactory perception and its implications for philosophical theories of the objects of olfactory perception can be found in Barwich (2016).

${ }^{24}$ The current section's argument and evidence derives from mappings and encodings of stimuli as inputted to the $P C$, which suggest the coarse grained distributed functionally compositional representations of complex odorants. However, the pathways between the $P C$ and the orbital frontal 
research has progressed our understanding of the $P C$ as an associative center for olfactory processing and as integral for generating configural odor objects from the molecular properties encoded at lower levels of sensory processing (for a review see Courtiol and Wilson, 2016).

While chemotopic organization is not maintained in the $P C$, it has been documented that cortical neurons within the dorsoposterior part of the anterior $P C$ in rats, display neural specificity to some food related categories of odorants, either in a holistic fashion to the entire mixture of odorants derived from the food source, or selectively to a prominent component of the mixture (Yoshida and Mori 2007). Despite cortical neurons within the PC displaying some manner of sensitivity to the odorant category, further research substantiates interpreting the $P C$ as an associative network for generating categorical stereotypes of odorants not in terms of their concatenative compositionality, but in a holistic fashion (Stettler and Axel, 2009). Barnes et al., (2008) found that neuron ensembles within the rat $P C$ treat an odorant composed of ten components as equivalent to that same mixture minus one component. Their results indicate that the $P C$ completes the representation of the complex odorant even in the absence of a component. Additionally, their research showed the neural ensembles discriminated between two different tencomponent mixtures. Together these studies suggest that the olfactory system at the $P C$ encodes odorant categories of varying molecular complexity in a combinatorial manner that does not depend upon explicitly representing each of the component parts. The combinatorial syntax of olfactory stimulus transduction within the $P C$ does not depend upon the explicit representation of each constituent in a manner required for concatenative compositionality. Thus, it is arguably the case that olfactory processing at the $\mathrm{PC}$ implements a system of functional compositionality that does not require access to all of the constituents or the decomposition of the complex mixture into their constituents.

\subsection{Olfactory Mixture Perception}

Further, evidence that olfactory system, and in particular perceptual states, implement nondecomposable functional compositionally can be derived from research on olfactory mixtures. Olfactory mixtures occur when two or more odorants are combined to form a complex odor. The majority of smells are composed of a vast number of molecular compounds. Consequently, the nature of olfactory mixtures is of great importance in attempting to understand how it is that we recognize, identify, and individuate a smell. There is evidence for distinct encoding at the cortical level between single and binary odor mixtures (Boyle et al., 2009). However, in what follows, the focus will be upon the encoding of odor mixtures, as these are the most decisive in examining the compositionality employed by the olfactory system.

When two or more odorants are combined into a complex, one of two possible mixtures results: a configural mixture whose olfactory quality differs from the smell of the components which are not discernable as constituents of the new smell; or an elemental mixture whose olfactory quality is merely the concatenation of the components that are discernable within the complex (Berglund et al., 1973). Configural mixtures are particularly

cortex (OFC) suggest that there is a degree of organization and spatial topography to odorant encoding within the $P C$ in connection with the $O F C$ (Chen et al., 2014). Since the issue at hand concerns the pathways relevant to odor language encoding these pathways between the PC and OFC will be left aside. Olofsson and Gottfried's research (see section 2.2.3) indicates that the projection to the ATL is from the PC, thus the role of the OFC in this regard is not clearly of relevance. 
fascinating since the mixture's quality is not determined as an additive process such that one can predict the new smell from its individual components (Berglund et al., 1973).

Initial research on rodents indicated that perceptually similar odorants (i.e. those with similar olfactory qualities) yield configural mixtures, while dissimilar odorants yield elemental mixtures (Wiltrout et al., 2003). However, it has since been shown that the resultant quality of an olfactory mixture is better accounted for by $O R N$ receptivity (Kay et al., 2003, 2005). Odorants formed by similar molecular structures activate similar sets of receptor neurons thereby generating configural mixtures, while those differing in structure yield elemental mixtures.

Additionally, Kay et al. (2005) uncovered the concentration effect that similar and dissimilar components can yield both kinds of mixtures depending upon the concentration levels of the constituents. By varying the concentration of odorant components, one can influence whether the complex mixture will be perceived as configural or elemental (McNamara et al., 2007). The concentration effect goes some way towards demonstrating that olfactory mixture quality is not a simple additive process, nor is it completely determined and predicted by the olfactory quality of the constituents. What becomes clear is that the olfactory system can represent complex olfactory stimuli either in a classical compositional manner (elemental mixtures) or employing functional compositionality (configural mixtures).

Recent work on olfactory processing has shown that the olfactory system employs two distinct centers for the neural realization of our perception of configural and elemental mixtures. Elemental olfactory mixtures are encoded within the Amygdala $(A M)$, while configural mixtures are encoded within the posterior $P C(P P C)$ (Howard and Gottfried, 2014). Hence, the cortical area activated when we have a configural olfactory experience is implicated in encoding odorants in a non-decompositional form of functional compositional, as discussed in section 4.1.2. The combinatorial syntax of transduction at the sensory level, in combination with distinct areas responsible for processing configural and elemental olfactory mixtures sheds light on the next olfactory phenomena of our inability to identify the components of olfactory mixtures, which suggests that olfactory employs nondecomposable functional compositionality. ${ }^{25}$

Livermore and Laing (1996, 1998) and Laing et al., (1989, 1992, 1999, 2001, 2002) documented our inability to identify odorants within a complex odor. They established that even if the subject had access to the individual constituent of the complex odor, they could identify at most three to four of the components within a complex chemical mixture. The inability to perceptually identify the constituents within a complex smell is best explained in light of the aforementioned evidence that the nature of sensory and cortical encoding of olfactory stimuli does not always encode complex odors as the concatenation of their constituents. The limitation in identifying parts of a complex odor might be attributed to the compositional format outputted from the $P C$.

The Laing effect and configural olfactory mixtures demonstrate that the constituents of complex olfactory perceptual states are not readily discernable in the manner that would be expected if olfaction implemented compositionality in the strong concatenative or functional

${ }^{25}$ Given the distinct pathways for configural and elemental mixtures together with the pathway known to be associated with connection to the language centers in the ATL transiting the PC, it cannot be argued that the olfactory system could simply output the atomistic representation of each odor we can discriminate into our conceptual system. 
decomposable manner. Our inability to identify more than four components of a complex olfactory mixture suggests that all of the constituents of the mixtures are not perceptually accessible. Since we can accurately estimate the concentration of the constituents and we experience the mixture as a complex entity that transcends the summation of its constituents, it cannot be objected that these are simply cases of the olfactory system generating new primitives.

Configural mixtures confirm that our olfactory perceptual abilities do not represent complex olfactory stimuli as the mere tokening of their constituents. Since we can manipulate the concentration effect in configural mixtures to shift the overshadowing of the components, as well as change them into elemental mixtures, it suggests that these perceptual states are representationally complex. Veridical experiences of these mixtures present a complex entity that is not merely the summation of its parts. Moreover, the constituents are not identifiable as distinct tokens within the complex stimuli. Configural olfactory mixtures are not represented in a concatenative or functionally decomposable fashion; rather the best explanation of their representational structure only requires the most minimal notion of compositionality.

\subsection{Olfactory Imagery}

Algom and Cain (1991) originally showed that olfactory imagery mimics the veridical perception of odor mixtures. Generally, mixtures of perceptually similar components are judged as less intense than would be expected if the intensity consisted of the summation of the parts. Subjects in their study generated the same imagistic percepts in accordance with this naturally occurring perceptual phenomenon. The far-ranging implication is that we can mentally combine mixtures with the same compositional results as those of actual olfactory mixtures. It is arguably the case that olfactory cognitive states also employ the same format of compositionality. Our capacity for olfactory imagery (reviewed in Stevenson et al., 2005; Arshamian and Larson, 2014; Young, 2014), which mimics veridical perception, suggests that the compositional representational format is preserved from sensory and cortical processing through perceptual and cognitive states. While the evidence covered throughout section 4 shows comprehensively across levels of processing and means of experimental methods that olfaction employs a different format of compositionality than that employed by semantically accessible concepts, as surveyed in section 3, the next section considers a possible strong line of criticism, which will be shown does not obviate the table thesis of the paper, but rather offers further reason to think considering the format and cortical realization of compositional olfactory states and linguistic semantic states is of importance in understanding the discrepancy between olfactory discrimination and identification.

\section{Possible Criticism: the discrepancy is culturally mediated and arises from linguistic practices.}

A possible line of criticism of the entire research project of explaining the discrepancy in identifying odors by names is to deny at the outset that there is a puzzling deficit that requires explanation. The discrepancy between olfactory capacities for identification and discrimination is not universal; rather it might reflect western culture's contempt for smell. English does not have a designated odor lexicon (Majid, 2015), but cultures with a robust lexicon for odors that is employed in everyday life display little to no discrepancy in identifying odors (for a brief review cf O’Meara and Majid, 2016). ${ }^{26}$ For instance, Maniq has

${ }^{26}$ Despite only showing $26.3 \%$ accuracy at odor naming a recent study by Hulsman and Majid (2018) suggests that in addition to odor familiarity (as indicated in introduction) the familiarity of 
a rich culture of talking about smells using a two-dimensional odor lexicon that does not extensionally refer to natural kinds but picks out common odor qualities. Additionally, the Aslian community of Jahai have a dedicated lexicon of olfactory terms and categories that are integral to their daily lives. Moreover, when compared to English speakers they show no deficit between their descriptive capacity for colors and odors (Majid and Burenhult, 2014). ${ }^{27}$ In a more recent study Majid and Kruspe (2018) go on to attribute the inability to name odors as a culturally contingent fact that is related to subsistence mode, such that it is not seen in their subset of Aslian communities that are hunter gathers, but it is seen within those whose subsistence depends more on agriculture and trade. Thus, the discrepancy is not a universal phenomenon, but partially dependent on language, culture, and mode of subsistence.

The cultural relativity of the accuracy of identifying odors might be taken as a counterexample to the thesis that the deficit for English speakers is attributable to an incompatibility in the representational format employed by the conceptual semantic systems and olfactory language centers. However, the lack of a discrepancy in some cultures does not negate that there is a puzzle when relativized to English speakers. Furthermore, noting that different lexicons generate different olfactory capacities strengthens the driving claim of the paper that compositional formats must also be taken into account. In fact, Olofsson and Gottfried (2105) and Majid (2015) agree that cortical connectivity on its own will not be sufficient to explain why discrepancies exist between cultural groups with different odor lexicons.

If we consider language as an ongoing innovation that effects the plasticity and neural architecture of the human brain, then odor lexicons might generate neural connectivity differences between cultures, which is noted as a possible explanation by Majid (2015), as well as Olofsson and Wilson (2018). Majid (2015) list a number of instances of languages effecting cortical connectivity. In particular, it has been shown that professional perfumers have increased density of cortical areas associated with olfactory processing indicating neural plasticity, change in connectivity, and neural reorganization for these olfactory experts (Delon-Martin, et al. 2013). Thus, the explanation on offer does not run contrary to cultural differences, but further supports the need to understand the underlying formative

the odor label also plays a modulating role in increasing naming accuracy (instrumentally measured as the descriptive content of responses). Thus, they conclude that odor label frequency also needs be taken into account to explain the difficulty in naming odors. Based on these results they suggest that more research needs to be conducted on the effect of lexical properties on our olfactory perceptual capacities, yet the thesis of this paper that the compositional format of representations (an arguably linguistic property when applied to our semantic conceptual resources) at each processing center within the olfactory system projecting to language centers is not considered.

27 As a methodological aside, the task within Majid and Burenhault (2014) and Majid and Kruspe (2018) is disanalogous to the odor identification task used in the studies reviewed in the introduction. The method employed in both these studies compares the description of the participant to the experimental cohort in terms of (a) descriptive agreement, (b) length of utterance, and (c) type of response. The descriptive analysis is not the same method employed in the studies of English speakers, which requires the participant to freely generate a veridical linguistic tag (name) for an odorant. However, that is not to question the validity of the cross-cultural comparison of Majid and Burenhault (2014), as their measure compared English to Jahai speakers on (a-c) for descriptions of colors and odors under the assumption that smells are qualities of objects and not objective perceptual entities (an assumption criticized by Olofsson and Gottfried, 2015). Further scrutiny of the Majid and Burenhault (2014) results indicate that while Jahai might not have a discrepancy between describing colors and odors, they lag well behind English speakers' capacity to describe colors, which might suggest they have greater cognitive and neural resources available for deployment in their use of an odor lexicon. 
nature of semantic conceptual representations and those employed by our olfactory language systems. Further research needs to be conducted on the neural representation of odors cross-culturally. For instance, do individuals in these cultures display the same inabilities in decomposing complex odors into their constitutive components (see section 4.2)? Additionally, it would be of further general interest to explore the compositional format of semantic concepts in these cultures with rich odor lexicons.

A further worry for the explanation on offer that the deficit in olfactory identification within western cultures might be attributed to an incompatibility in representational formats is the claim that people can improve their capacity to name odors in a short period of time through training. Cain (1979) claims to have shown an increase from 60\% accuracy to $77 \%$ over the course of four sessions spaced two days apart. However, in the re-testing session the task re-used the linguistic labels from the previous session, thereby generating a measure of matching and recognition across trials and not the self-generated naming task that was used in the initial conditions. Similarly, Sulmont-Rosse et al (2005) show that if individuals re-use their self-generated linguistic labels across trials, their odorant recognition increases, but this does not demonstrate an increase in odor identification. Directly contradicting Cain's (1979) finding, Jehl et al. (1997) showed that verbal encoding enhances the encoding of odorants for short-term and long-term memory in a manner that increases detection and recognition, yet identification decreased across trials.

While it is plausible that increasing our odor lexicon and verbal descriptions of smells might increase olfactory identification, even highly trained wine and coffee experts whose livelihood depends upon their use of specialized odor lexicons do not show increased accuracy in olfactory identification (Croijmans \& Maid, 2016). Since the aforementioned studies do not show an increase in identification across trials, it might be wondered how odorant encoding and memory can be increased across time? Lyman and McDaniels (1990) show that odorant encoding is improved when both verbal/linguistic and visual routes are also used to encode an odorant. Employing two extra encoding methods increases retrieval rates, making it likely that as odorant encoding converts more resources from vision and semantic centers, odorant encoding and memory is improved. Improvements in odorant recognition and identification might be attributed to converting resources from visual cortical processing, which might sound implausible based on the speed of neural plasticity this would require. However, Qu et al. (2016) show neural plasticity within two days of learning de novo odor-visual categories that require the use of both olfactory and visual cues. All indications from the experimental literature are that linguistic prowess does help for discrimination, detection, and recognition, but from the outset it has been noted that we are gifted in this regard. Moreover, in those instances of cross-cultural comparison that do not show a deficit between olfactory capacities for discrimination and identification, the theorists involved in the debate agree that linguistic usage can effect cortical connectivity based on the format and use of language, thereby making room for the thesis of the paper that explains the discrepancy based on the different compositional formats employed by olfaction and our conceptual semantic resources.

\section{Conclusion}

Our initial puzzle was why is it that we are gifted in our olfactory abilities for detecting and discriminating odorants, yet pitiful at identifying odors by name? The discrepancy involves accessing and deploying semantic information, since identifying an odorant requires the deployment of our semantic conceptual resources, while detection and discrimination does not. The most likely explanation given this line of thought, is that the problem arises as an interface issue between conceptual resources and olfactory processing, since it is not 
obvious that detection and discrimination require conceptual resources, but odor identification does. Also, as noted in the introduction, in those situations that we cannot identify the odor, we also do not have access to associated semantic information about the smell. The thesis argued for in this paper is that the problem is not just a lack of connectivity or the amount of stimulus processing before the olfactory system connects to cortical language centers. Rather, the olfactory system employs a compositional format that is incompatible with the representational nature of our conceptual semantic resources.

The format of stimuli transduction and encoding from sensory to cortical areas in the olfactory system as it projects to olfactory language centers does not require the explicit representation of each constituent within the complex representation, yielding a form of functional compositionality that does not readily allow the complex to be decomposed into its constituents, which facilitates efficient detection and discrimination, but not identification. The format of olfactory stimuli transduction and encoding allows for an extremely efficient representational system that is sensitive to the vast range of variegated chemical stimuli (including complex mixtures) that we can detect and discriminate.

The reason we cannot identify odorants with ease is because our conceptual semantic resources operate using a hierarchical system of representation that is either concatenatively compositional or able to access the constituents using a decomposition function, while the olfactory system employs a more minimal form of compositionality. Even in those instances that our experience of smells yields olfactory mixtures with an internal syntax reminiscent of classical compositionality, these are encoded by the amygdala, which is not directly connected to olfactory language centers. The language centers of olfaction do not represent or parse the incoming projections from the piriform cortex in a manner that enables it to be readily employed by our conceptual semantic processing hubs. The modality specific area of the cortex responsible for olfactory language convergence, the ATL, receives its input from the piriform cortex, which does not obey the strictures of classical compositionality or even decomposable functional compositionality. Moreover, the ATL has been associated with processing the syntactic aspects of compositional linguistic representation, but it is not implicated in processing compositional semantics. Future empirical research is required to support the thesis of this paper to fully asses the compositional format employed within the ATL for olfactory processing, as well as the compositional format of neural processing for those cultures that have a rich odor lexicon. However, explaining the puzzling discrepancy as an interface issue between incompatible compositional formats allows an integrated explanation across each stage of olfactory processing, as well as providing a unified explanation of why we are gifted at olfactory discrimination, yet bad at identifying smells.

\section{References:}

Algom, D., Cain, W.S. 1991: Remembered odors and mental mixtures: Tapping reservoirs of olfactory knowledge, Journal of Experimental Psychology: Human Perception Eं Performance, pp. 1104-1119.

Amoore. J. 1970: Molecular Basis of Odor, Charles C. Thomas, Springfield, IL.

Araneda, R. C., Kini, A. D., and Firestein, S. 2000. The molecular receptive range of an odorant receptor, Nature Neuroscience, vol. 3,no.12, pp. 1248-1255.

Arshamian A and Larsson M. 2014. Same same but different: the case of olfactory imagery. Front. Psychol. 5:34. doi: 10.3389/fpsyg.2014.00034 
Auffarth, B. 2013. Understanding smell - the olfactory stimulus problem. Neuroscience and Biobehavioral Reviews, 37:8, 1667-1679.

Barnes, D. C., Hofacer, R. D., Zaman, A. R., Rennaker, R. L., \& Wilson, D. A. (2008).

Olfactory perceptual stability and discrimination. Nature Neuroscience, 11(12), 1378-1380.

doi: $10.1038 / \mathrm{nn} .2217$

Batty, C.E. 2009: 'What's that Smell?', Southern Journal of Philosophy 27: 32 1-348.

—2010a: 'What the Nose Doesn't Know: Non-Veridicality in Olfactory Experience', Journal of Consciousness Studies 17: 10-17.

—2010b: 'Scents and Sensibilia', American Philosophical Quarterly 47(2): 103118.

—2010c: 'A Representational Account of Olfactory Experience', Canadian Journal of Philosophy 40(4): 511-538.

Barker, C. \& Jacobson, P. (eds) 2007: Direct Compositionality. Oxford University Press

Barwich, Ann-Sophie (2014). A Sense So Rare: Measuring Olfactory Experiences and Making a Case for a Process Perspective on Sensory Perception. Biological Theory 9 (3):258-268.

2015. Bending Molecules or Bending the Rules? The

Application of Theoretical Models in Fragrance Chemistry. Perspectives on Science 23 (4):443-465.

—2016. "Making Sense of Smell" The Philosophers' Magazine 73, 41-47.

—2017. "Measuring the World: Towards a Process Model of Perception," in: Everything Flows:

Towards a Processual Philosophy of Biology, ed. by D. Nicholson, and J. Dupré. Oxford University Press.

Bensafi, M. et al (2014) The effect of verbal context on olfactory neural responses. Human Brain Mapping 35:810-818

Berglund, B., et al.,.,, 1973: 'A Quantitative Principle of Perceived Intensity Summation in Odor Mixtures', Journal of Experimental Psychology 100(1):29-38.

Bermudez, J.L. 2007: 'What is at stake in the debate on non-conceptual content?', Philosophical Perspectives 21: 55-72.

Binder, J. R., J. A. Frost, et al. 1997: "Human Brain Language Areas Identified by Functional Magnetic Resonance Imaging." The Journal of Neuroscience 17(1): 353-362.

Binder, J.R. 2015: In defense of abstract conceptual representations. Psychon Bull

Rev 23:1096-1108 DOI 10.3758/s13423-015-0909-1

Boyle, JA, Djordjevic, J., Olsson, MJ., Lundström, JN., and Jones-Gotman , M. 2009: The Human Brain Distinguishes between Single Odorants and Binary Mixtures. Cereb. Cortex 19 (1): 6671 doi: $10.1093 /$ cercor/bhn058

Bozza, T.C., and Mombaerts, P. 2001: 'Olfactory coding: Revealing intrinsic representations of odors', Current Biology 11(17): R687-R690.

Broome, B., M., Jayaraman, V., \& Laurent, G. 2006: 'Encoding and Decoding of Overlapping Odor Sequences', Neuron 51: 467-482. 
Buck, L.B., and Axel, R. 1991: 'A novel multigene family may encode odorant receptors: a molecular basis for odor recognition', Cell 65/; 175-189.

Bushdid, C., Magnasco, M.O., Vosshall, L.B., and Keller, A. 2014: Humans can discriminate more than 1 trillion olfactory stimuli. Science $343,1370-1372$.

Cain, W.S. 1979: Know with the nose - keys to odor identification. Science 203, 467-470

Cain, W.S. et al (1995) Life-span development of odor identification, learning, and olfactory sensitivity. Perception 24: 1457-1472.

Castro, J. B. and Seeley, W. P. 2014: "Olfaction, valuation, and action: Reorienting perception." Frontiers in Psychology 5.

Chen, C., Zou, D., Altomare, C.G., Xu, L., Greer, C.A., and Firestein, S.J. 2014. Nonsensory targetdependent organization of piriform cortex. PNAS 111 (47) 16931-16936, doi: $10.1073 /$ pnas.1411266111

Clapp, L. 2012: Is even thought compositional? Philosophical Studies 157 (2):299-322.

Clarke A, Taylor KI, Devereux B, Randall B, Tyler LK. 2013. From per- ception to conception: how meaningful objects are processed over time. Cereb Cortex. 23:187-197.

Clarke A, Tyler LK. 2014. Object-specific semantic coding in human perirhinal cortex. J Neurosci. 34(14):4766-4775.

Clarke, A., Devereux, B.J., Randall B. and Tyler, L.K. 2015. Predicting the Time Course of Individual Objects with MEG. Cerebral Cortex;25:3602-3612 doi:10.1093/cercor/bhu203

Connell, L. and Lynott, D. 2014. Principles of Representation: Why You Can't Represent the Same Concept Twice. Topics in Cognitive Science 6 390-406

Courtiol, E. and Wilson, D.A. 2016. Neural Representation of Odor-Guided Behavior in the Rat Olfactory Thalamus. Journal of Neuroscience 36 (22) 5946-5960; DOI: 10.1523/JNEUROSCI.053316.2016

Crane, T. 1990: The language of thought: no syntax without sematics. Mind $\mathcal{E}^{2}$ Language, 5, 187212.

Croijmans I, Majid A (2016) Not All Flavor Expertise Is Equal: The Language of Wine and Coffee Experts. PLoS ONE 11(6): e0155845. doi:10.1371/journal.pone.0155845

Crosson, B. 2012. Thalamic mechanisms in language: A reconsideration based on recent findings and concepts. Brain \& Language 126 (2013) 73-88

Cummins, R. 1996:. Systematicity. The Journal of Philosophy. 93, 591-614.

Cummins, R., Blackmon, J. Byrd, D., et. all 2001: Systematicity and the congition of structured domains. The Journal of Philosophy. 98, 167-1 85.

Del Pinal, G. 2015. Prototypes as compositional components of concepts. Synthese DOI $10.1007 / \mathrm{s} 11229-015-0892-0$

Delon-Martin, C. et al. 2013. Perfumers' expertise induces structural reorganization in olfactory brain regions. NeuroImage 68: 55-62 
Dever, J. 2006: “Compositionality,” In Ernest Lepore \& Barry Smith (eds.), The

Oxford Handbook of Philosophy of Language. Oxford University Press. 633--666

—2012: "Compositionality", in The Routledge Handbook to the Philosophy of

Language, 91-102.

Earley, J. E. 2005: 'Why There is No Salt in the Sea', Foundations of Chemistry 7: 85-102.

Engen, T. and Ross, B.M. (1973) Long-term memory of odors with and without verbal descriptors.

J. Exp. Psychol. 100, 221-227

Evans, G. 1982: The Varieties of Reference. J. McDowell (ed.), Clarendon Press, Oxford, U.K..

Frank, R.A., et al. 2011. Odor Recognition Memory as a Function of Odor-Naming

Performance. Chem. Senses 36: 29-41.

Firestein, S. 2001. "How the olfactory system makes sense of scents," Nature,vol.

413, no. 6852, pp. 211-218.

Fodor, J.A. 1976: The Language of Thought. Hassocks, Sussex, UK: The Harvester Press.

-1981: 'Methodological Solipsism Considered as a Research

Strategy in Cognitive Psychology', in: J. Fodor, Representations, The Harvester Press, Sussex, Brighton, UK: 225-253.

-1981: The Current Status of the Innateness Controversy. In Representations. Cambridge, MA: MIT Press.

—1987: Psychosemantics. Cambridge, Mass.: MIT Press.

—1985: 'Fodor's guide to mental representations: the intelligent Auntie's Vade-

Mecum', A Theory of Content and Other Essays, MIT Press, Cambridge, MA.

—1998: Concepts, Oxford University Press, Oxford, U.K.

—2000: The mind doesn't work that way, The MIT Press, Cambridge, MA.

—2001: "Language, Thought, and Compositionality," Mind and Language,16: 1-15.

—2008: LOT2, Oxford University Press, Oxford, U.K.

Fodor, J. A., and Z. Pylyshyn. 1988: Connectionism and Cognitive Architecture: A Critical Analysis. In Connections and Symbols, edited by S. Pinker and J. Mehler. Cambridge, MA: MIT Press.

Fodor, J. A., and E. Lepore. 1996: The Red Herring and the Pet Fish: Why Concepts Still Can't be Prototypes. Cognition 58:253-270.

Fodor, J. \& McLaughlin, B.P. 1990: 'Connectionism and the problem of Systematicity', Cognition 35: 83-204.

Frederick, D.E., \& Korsching, S. I. 1997: 'Combinatorial and Chemotopic Odorant Coding in the Zebrafish Olfactory Bulb Visualized by Optical Imaging', Neuron 18: 737-752.

Frederick, D.E., et al. 2009: 'A Critical Test of the Overlap Hypothesis for Odor Mixture Perception,. Behavioral Neuroscience 123(2): 430-437.

Frater, G., Bajgrowicz, J.A. \& Kraft, P. (1998), ‘Fragrance chemistry’,Tetrahedron 54: 7633-7703. 
Frixione, M. and Lieto, A. 2012. Representing Concepts in Formal Ontologies. Logic and Logical Philosophy 21, 391-414 DOI: 10.12775/LLP.2012.018

Gerkin, R.C. and Castro, J.B. 2015. The number of olfactory stimuli that humans can discriminate is still unknown. eLife; 4 DOI: $10.7554 /$ eLife.08127

Gilbert, A. N. 2008: What the nose knows. New York: Crown Publishers.

Goodman, N., Tenenbaum, J., \& Gerstenberg, T. (2015). Concepts in a Sciences probabilistic language of thought. In E. Margolis \& S. Laurence (Eds.) The conceptual mind: New directions in the study of concepts (623-655). Cambridge, MA: MIT Press.

Hallem EA, Carlson JR. 2006a. Coding of odors by a receptor repertoire. Cell 125: $143-160$.

Hallem EA, Dahanukar A, Carlson JR. 2006b Insect odor and taste receptors. Annu Rev Entomol 51: 113-135.

Herz, R. S. 2005: The unique interaction between language and olfactory perception and cognition. Trends in Experimental Psychology Research. New York, Nova Science Publishers, Inc.: 91-10

Hildebrand, J.G., \& Shepherd, G.M. 1997: 'Mechanism of olfactory discrimination', Annual Review of Neuroscience 20: 595-631.

Homewood, J. and Stevenson, R.J. 2001 Differences in naming accuracy of odors presented to the left and right nostrils. Biological Psychology 58: 65-73

Horgan, T. \& Tienson, J. 1994: A nonclassical framework for cognitive science. Synthese, 101, 305345 .

Horgan, T., \& Tienson, J. 1996: Connectionism and the Philosophy of Psychology. Cambridge, MA: The MIT Press.

Howard, JD. and Gottfried, JA. 2014: Configural and Elemental Coding of Natural Odor Mixture Components in the Human Brain. Neuron, 84 (4): 857 - 869.

Humphries, C. Binder, J.R., Medler, D.A. and Liebenthal, E. 2006. Syntactic and Semantic Modulation of Neural Activity during Auditory Sentence Comprehension. Journal of Cognitive Neuroscience 18:4, pp. 665-679

Huisman, JLA and Majid, A. 2018. Psycholinguistic variables matter in odor naming, Memory \& Cognition https://doi.org/10.3758/s13421-017-0785-1

Illig KR, Haberly LB (2003) Odor-evoked activity is spatially distributed in piriform cortex. J Comp Neurol 457(4):361-373.

Jacobson, P. 2002: 'The (Dis)organisation of the Grammar: 25 years'. In: Linguistics $\&$ Philosophy 25, pp. 601-26.

Jehl, C. et al. 1997. Role of verbal encoding in short- and long-term odor recognition. Perception and Psychophysics, 59 (1), 100-110.

Jönsson, F. U., Tchekhova, A., et al. 2005: A metamemory perspective on odor naming and identification. Chemical Senses, 30(4), 353-365. 
Jönsson, F. U., Olosson, H., and Olsson, M.J. 2005. Odor Emotionally Affects the Confidence in Odor Naming. Chem. Senses 30: 29-35.

Jylkka, J. 2011. Hybrid Extensional Prototype Compositionality. Minds \& Machines 21:41-56 DOI $10.1007 /$ s 1 1023-010-9217-8

Kay, L. M., Crk, T., \& Thorngate, J. 2005: 'A Redefinition of Odor Mixture Quality', Behavioral Neuroscience 119(3): 726-733.

Kay, L.M., Lowry, C.A., \& Jacobs, H.A. 2003: 'Receptor Contributions to Configural and Elemental Odor Mixture Perception', Behavioral Neuroscience 117(5): 1108-1114.

Keller, A. 2016. Philosophy of Olfactory Perception. Palgrave Macmillan

- 2017. Philosophy of Olfactory Perception. Palgrave Macmillan

Kiefer, M. and Pulvermuller, F. 2012. Conceptual representations in mind and brain: Theoretical developments, current evidence and future directions. Cortex 48:7 805-825.

Klostermann, Fabian, Krugel, and Ehlen. 2013. Functional roles of the thalamus for language capacities. Frontiers in Systems Neuroscience 7.

Laing, D.G., \& Francis G.W. 1989: 'The capacity of humans to identify odors in mixtures', Physiology and Behavior 46(5): 809-814.

Laing, D.G., \& Glemarec, A. 1992: 'Selective attention and the perceptual analysis of odor mixtures', Physiology and Behavior 52: 1047-1053.

Laing, D.G., \& Jinks A. 1999: 'Odor identification in mixtures: is olfactory working memory the ultimate limitation?’, Chemical Senses 24(5): 583.

Laing, D.G., Jinks, A., Link, C., \& Hutchinson, I. 2001: 'The capacity of humans to analyse odor mixtures and taste mixtures is limited by working memory', Chemical Senses 26(6): 702.

Laing, D.G., Link, C., Jinks, A.L., \& Hutchinson I. 2002: 'The limited capacity of humans to identify the components of taste mixtures and taste-odor mixtures', Perception 31(5): 617-635.

Lambon Ralph MA, Sage K, Jones RW, Mayberry EJ. 2010. Coherent concepts are computed in the anterior temporal lobes. Proc Natl Acad Sci USA. 107:2717-2722.

Laurent, G., Stopfer, M., Friedrich, R.W., Rabinovich, M.I.. Volkovskii, A. et al.,.,, 2001: 'Odor Encoding as an Active, Dynamic Process: Experiments, Computaton, and Theory', Annual Review of Neuroscience 24: 263-297.

Linster, C., and Cleland, T. A. (2013). "Spatiotemporal coding in the olfactory system," in 20 Years of Computational Neuroscience, ed J. M. Bower (New York, NY: Springer New York), 229-242. doi: 10.1007/978-1-4614-1424-7_11

Livermore, A., \& Laing, D.G. 1996: 'Influence of training and experience on the perception of multicomponent odor mixtures', Journal of Experimental Psychology: Human Perception and Performance 22: 267-277.

—1998: 'The influence of odor type on the discrimina- tion and identification of odorants in multicomponent odor mixtures', Physiology and Behavior 65(2): 311-320.

Lorig, T. S. 1989: "Human EEG and odor response." Progress in Neurobiology 33(5-6): 387-398. 
Lorig, T. S., D. G. Elmes, et al. 1998: "Chemosensory alteration of information processing." Annals of the New York Academy of Sciences 855: 591-597.

Lorig, T. S. 1999: "On the similarity of odor and language perception." Neuroscience E Biobehavioral Reviews 23(3): 391-398.

Lycan WG. 2014: The intentionality of smell. Front. Psychol. 5:436. doi: 10.3389/fpsyg.2014.00436

-2017. What does taste represent. Australasian Jounral of Philosophy. doi.org/10.1080/00048402.2017.1291697

Marshall, W.H., Woolsey, C.N., and Bard, P. 1941: Observations on cortical somatic sensory mechanisms of cat and monkey. J. Neurophysiol. 4, 1-24.

Martinez-Manrique, Fernando 2014. Systematicity and Conceptual Pluralism. In Paco

Calvo John Symons (ed.), The Architecture of Cognition: Rethinking Fodor and Pylyshyn's Systematicity Challenge. MIT Press. pp. 305-334.

Majid \& Kruspe, 2018. Hunter-Gatherer Olfaction Is Special. Current Biology 28, 409-413.

Majid, A., Speed, L., Croijmans, I., \& Arshamian, A. 2017. What makes a better smeller? Perception, 46, 406-430. doi:10.1177/0301006616688224.

Majid, A. 2015. Cultural factors shape olfactory language. Trends in Cognitive Sciences, 19(11), 629-630. doi:10.1016/j.tics.2015.06.009

Majid, A., \& Burenhult, N. 2014. Odors are expressible in language, as long as you speak the right language. Cognition, 130(2), 266-270. doi:10.1016/j.cognition.2013.11.004

Margolis, Eric \& Laurence, Stephen (eds.) (1999). Concepts: Core Readings. MIT Press.

Maye, A. and Engel, A.K. 2012. Neuronal assembly models of compostionality. In M. Werning, W. Hinzen, \& E. Machery (Eds.), The Oxford handbook of compositionality. Oxford: Oxford University Press.

McGann, J.P. 2017. Poor human olfaction is a 19th century myth. Science 356:eaam7263

McNamara, A. M., Magidson, P.D., \& Linster, C. 2007: 'Binary Mixture Perception is Affected by Concentration of Odor Components', Behavioral Neuroscience 121(5): 1132-1136.

Meierhenrich, U.J., Golebiowski, J., Fernandez, X., and Cabrol-Bass, D. 2004. The molecular basis of olfactory chemoreception, AngewandteChemie,vol.43,no.47,pp.6410-6412.

Meister, M. (2015) On the dimensionality of odor space. eLife 2015;4:e07865 DOI: $10.7554 /$ eLife.07865

Mion M, Patterson K, Acosta-Cabronero J, Pengas G, Izquierdo-Garcia D, Hong Y, Fryer T, Williams G, Hodges J, Nestor PJ. 2010: What the left and right anterior fusiform gyri tell us about semantic memory. Brain. 133:3256-3268.

Mori, K., \& Shepherd, G.M. 1994: 'Emerging principles of molecular signal processing by mitral/tufted cells in the olfactory bulb', Cell Biology 5: 65-74.

Moss HE, Rodd JM, Stamatakis EA, Bright P, Tyler LK. 2005. Anterome- dial temporal 
cortex supports fine-grained differentiation among objects. Cereb Cortex. 15:616-627.

Murphy, G.L. 2002. The Big Book of Concepts. MIT Press.

Olofsson JK 2014: Time to smell: a cascade model of human olfactory perception based on response-time (RT) measurement. Front. Psychol. 5:33. doi: 10.3389/fpsyg.2014.00033

Olofsson, J.K., Rogalski, E., Harrison, T., Mesulam, MM, \& Gottfried, J.A. 2013: A cortical pathway to olfactory naming: evidence from primary progressive aphasia. Brain 136: 12451259.

Olofsson JK, Hurley RS, Bowman NE, Bao X, Mesulam MM, Gottfried JA. 2014: A designated odor-language integration system in the human brain. J Neurosci. 2014 Nov 5;34(45):14864-73. doi: 10.1523/JNEUROSCI.2247-14.2014.

Olofsson, J.K. and Gottfried, J.A. 2015. The muted sense: neurocognitive limitations of olfactory language. Trends in Cognitive Science, 19:5.

Olofsson, J.K. and Gottfried, J.A. 2015. Response to Majid: Neurocognitive and Cultural Approaches to Odor Naming are Complementary . Trends in Cognitive Science, 19:1 1, 630.

Olofsson, J.K. and Wilson, D.A. 2018. Human Olfaction: It Takes Two Villages. Current Biology 28, R103-R126,

O'Meara, C., \& Majid, A. (2016). How changing lifestyles impact Seri smellscapes and smell language. Anthropological Linguistics, 58(2), 107-131. doi:10.1353/anl.2016.0024

Pagin, P. and Westerståhl, D. 2010a: Compositionality I: Definitions and Variants, Philosophy Compass 5:265-82.

Pagin, P. and Westerståhl, D. 2010b: Compositionality II: Arguments and Problems, Philosophy Compass 5:250-64.

Pauling, L. 1946: 'Molecular architecture and biological reactions', Biological

Sciences 24: 1375-1377.

Patterson K, Nestor PJ, Rogers TT. 2007. Where do you know what you know? The representation of semantic knowledge in the human brain. Nat Rev Neurosci. 8:976-988.

Piantadosi, S., Tenenbaum, J.B., and Goodman, N.D 2016. Psychological Revierw, Vol. 123, No. 4, 392424

Price AR, McAdams H, Grossman M, Hamilton RH 2015a: A meta- analysis of transcranial direct current stimulation studies examining the reliability of effects on language measures. Brain Stimul 8:1093-1 100. CrossRef Medline

Price AR, Bonner MF, Peelle JE, Grossman M 2015b: Converging evidence for the neuroanatomic basis of combinatorial semantics in the angular gyrus. J Neurosci 35:3276 -3284. CrossRef Medline

Price AR, Peelle, J.E., Bonner MF, Grossman M, Hamilton RH 2016. Causal Evidence for a Mechanism of Semantic Integration in the Angular Gyrus as Revealed by High-Definition Transcranial Direct Current Stimulation. J Neurosci 6(13):3829 -3838

Prinz, J. 2012: Regaining composure: A defense of prototype compositionality. In M. Werning, W. Hinzen, \& E. Machery (Eds.), The Oxford handbook of compositionality. Oxford: Oxford University Press. 
— 2002. Furnishing the mind: Concepts and their perceptual basis. Cambridge, MA: MIT Press.

Pylyshyn, Z. 2003: 'Return of the mental image: are there really pictures in the brain?', Trends in Cognitive Sciences 7(3): 113-118.

—2006: Seeing and Visualizing. Cambridge MA: MIT Press.

Qu, L.P. et al. 2016. De Novo Emergence of Odor Category Representations in the Human Brain. The Journal of Neuroscience, 36(2):468 - 478.

Rabinovich, M., Huerta, R., \& Laurent, G. 2008: 'Transient Dynamics for Neural Processing', Science $321(5885)$ : 48-50.

Rennaker RL, Chen CF, Ruyle AM, Sloan AM, Wilson DA (2007) Spatial and temporal distribution of odorant-evoked activity in the piriform cortex. J Neurosci 27(7): 1534-1542.

Rice, C. 2013. Concept empiricism, content, and compositionality. Philos Stud 162:567-583. DOI 10.1007/s11098-011-9782-6

Rossiter, K. J. 1996: 'Structure-odor relationships', Chemical Reviews 96: 3201-3240

Royet, J. P. and Plailly, J. 2004: "Lateralization of olfactory processes." Chemical Senses 29(8): 731-745.

Royet, J.P. et al. 2004. Levels-of-Processing Effects on a Task of Olfactory Naming. Perceptual and Motor Skills, 98, 197-213.

Sanders, H., B.E. Kolterman, R. Shusterman, D. Rinberg, A. Koulakov, and J. Lisman. A network that performs brute-force conversion of a temporal sequence to a spatial pattern: relevance to odor recognition. Front Comput Neurosci, 8, 108

Savic, I. and Berguland, H. 2004. Passive Perception of Odors and Semantic Circuits. Human Brain Mapping, 21: 271-278.

Sela L., and Sobel N. 2010: Human olfaction: a constant state of change-blindness. Exp. Brain Res. 205, 13-2910.1007/s00221-010-2348-6

Seubert, J. et al (2013) Statistical localization of human olfactory cortex, NeuroImage 66, pages 333342

Sezille, C. Ferdenzi, A. Chakirian, A. Fournel, M. Thevenet, J. Gerber, T. Hummel, M.

Bensafi, Dissociated neural representations induced by complex and simple odorant molecules, Neuroscience, Volume 287, 26 February 2015, Pages 23-31, ISSN 0306-4522, http://dx.doi.org/10.1016/j.neuroscience.2014.12.011.

Shepherd GM. 2004: The human sense of smell: are we better than we think? PLoS Biol 2:E146

-2005: 'Outline of a Theory of Olfactory Processing and its Relevance to Humans', Chemical Senses 39(S1): i3-i5.

-2009: An initial evaluation of the functions of human olfaction. Chem Senses 35:3-20

- 2012. Neurogastronomy. Columbia University Pres 
Smith, B.C. 2007. The Objectivity of Tastes and Tasting. In Questions of Taste: the philosophy of wine. Oxford University Press.

Stettler DD, and Axel, R. 2009: Representations of odor in the piriform cortex. Neuron 63:854-864

Stevenson, R.J. 2009: 'An initial evaluation of the functions of human olfaction', Chemical Senses 35(1): 3-20

Stevenson, R.J., \& Case, T.I. 2005: 'Olfactory imagery: A review', Psychonomic Bulletin $\Xi^{\circledR}$ Review 12(2): 244-264.

Stevenson, R.J., and Mahmut, M.K. 2013. The accessibility of semantic knowledge for odours that can and cannot be named. THE QUARTERLY JOURNAL OF EXPERIMENTAL

PSYCHOLOGY, 66:7, 1414-1431.

Stewart, T. and Eliasmith, C. 2012. Compositionality and biologically plausible models. In M. Werning, W. Hinzen, \& E. Machery (Eds.), The Oxford handbook of compositionality. Oxford: Oxford University Press.

Stopfer, M., Jayarman, V., \& Laurent, G. 2003: 'Intensity versus Identity Coding in an Olfactory System', Neuron 39: 991-1004.

Sulmont-Rosse, C. 2005: Odor naming methodology: Correct identification with multiple-choice versus repeatable identification in a free task. Chemical Senses, 30(1), 23-27.

Szabo, Z.G. 2000a: Compositionality as supervenience. Linguistics and Philosophy, 23:475-505.

—200ob: Problems of Compositionality. Garland Publishing, Inc

Talbot, S.A., and Marshall, W.H. 1941: Physiological studies on neural mechanisms of visual localization and discrimination. Am. J. Ophthalmol. 24, 1255- 1263.

Theofanopoulou. C. \& Boeckx, C. 2016: The central role of the thalamus in language and cognition. In Advances in Biolinguistics: The Human Language Faculty and its Biological Basis, Publisher: London: Routledge, Editors: Boeckx, Fujita

Tyler LK, Stamatakis EA, Bright P, Acres K, Abdallah S, Rodd JM, Moss HE. 2004. Processing objects at different levels of specificity. J Cogn Neurosci. 16:351-362.

Turin, L. 1996: ‘A Spectroscopic Mechanism for Primary Olfactory Reception', Chemical Senses 21(6): 773-791.

—2002: 'A Method for the calculation of odor character from molecular structure'. Journal of theoretical biology 216: 367-385.

—2006: The secret of scent: adventures in perfume and the science of smell, HarperCollins, New York, NY.

Turin, L. \& Yoshii, F. 2002: 'Structure-odor relations: a modern perspective', in: R. Doty (ed.), Handbook of Olfaction and Gustation Marcel Dekker, New York, NY.

van Gelder, T. 1990: Compositionality: a connectionist variation on a classical theme. Cognitive Science, 14, 335-384. 
Veramendi, M., Herencia, P., et al. 2013: Perfume odor categorization. Journal of Sensory Studies, $28,76-89$.

Wijk, R.A. and Cain, W.S. 1994. Odor Identification by Name and by Edibility. Human Factors, 36(1) 182-187.

Wiltrout, C., Dogra, S., \& Linster, C. 2003: 'Configurational and Nonconfigurational Interactions Between Odorants in Binary Mixtures', Behavioral Neuroscience 117(2): 236-245.

Woolsey, C.N., and Walzl, E.M. 1942: Topical projection of nerve fibers from local regions of the cochlea to the cerebral cortex of the cat. Bull. Johns Hop- kins Hosp. 71, 315-344.

Wnuk, E. and Majid, A. 2014. Revisiting the limits of language: The odor lexicon of Maniq. Cognition 131, 125-138.

Yaksi, E., von Saint Paul, F., Niessing, J., Bundschuh, S.T. \& Friedrich, R.W. 2009: 'Transformation of odor representations in target areas of the olfactory bulb', Nature Neuroscience 12(4): 474-482.

Yeshurun Y, Sobel, N. 2010: An odor is not worth a thousand words: from multidimensional odors to unidimensional odor objects. Annu Rev Psychol 61:(219-241):C211C2 15

Young, B.D. 2016. Smelling Matter. Philosophical Psychology. doi: 10.1080/09515089.2015.1126814

—2015: Formative Non-Conceptual Content, Journal of Consciousness Studies, 22.

—2014: Smelling phenomenal. Front. Psychol. 5:713. doi:

10.3389/fpsyg.2014.00713

—2012. Stinking consciousness! J. Conscious. Studies 19, 223-243.

-2011: Olfaction: Smelling the Content of Consciousness. Doctoral Dissertation, City University of New York, The Graduate Center.

Young BD, Keller A and Rosenthal D. 2014: Quality-space theory in olfaction.

Front. Psychol. 5:1. doi: 10.3389/fpsyg.2014.00001

Zelano, C. and Sobel, N. 2005: Humans as an animal model for systems- level organization of olfaction. Neuron 48:431-454

Zou, Z., \& Buck, L.B. 2006: 'Combinatorial Effects of Odorant Mixes in the Olfactory Cortex', Science 311: 1477-1482. 